\title{
Signal Processing For Tree-Trunk Investigation Using Ground Penetrating Radar
}

\author{
Iraklis Giannakis, Fabio Tosti, Livia Lantini, Daniel Egyir, Amir M. Alani \\ School of Computing and Engineering \\ University of West London (UWL) \\ London, United Kingdom \\ Iraklis.Giannakis@uwl.ac.uk, Fabio.Tosti@uwl.ac.uk, Livia.Lantini@uwl.ac.uk, Daniel.Egyir@uwl.ac.uk, Amir.Alani@uwl.ac.uk
}

\begin{abstract}
Invasive fungi diseases are considered one the biggest threats for the ash and oak forests in United Kingdom. To that extend, Ground Penetrating Radar (GPR) can provide a powerful diagnostic tool for assessing the health status of tree trunks based on their internal dielectric distribution. GPR acquisitions in tree-trunks is a unique problem that can not be approached with traditional GPR processing approaches. Typical interpretation tools like hyperbola fitting and migration should be adjusted and fine-tuned in order to be applicable for irregular measurements in a closed curve. The purpose of this paper is to provide GPR practitioners with a set of interpretation tools that can be applied in the field using commercial GPR antennas. In that context, a novel processing framework is presented that is fine-tuned for the current problem. The suggested scheme is successfully tested using both numerical and real data indicating the capabilities of GPR as a diagnostic tool for early detection of tree diseases.
\end{abstract}

Index Terms-Ash dieback, acute oak decline, AOD, forestry, ground penetrating radar (GPR), signal processing, tree.

\section{INTRODUCTION}

The importance of forests in the ecosystem and overall climate has been repeatedly highlighted in the literature [1], [2]. To that extend, the European Commission has published a green paper emphasizing on the importance of managing, monitoring and protecting European's forests [3]. One of the biggest threats for European's forests are invasive insects and diseases [2]-[4]. This became apparent in 1970s during the outbreak of elm disease in North America and Europe that brought the species Castanea dentata and Ulmus to an almost complete extinction [2], [5]. Currently, the fungi that cause ash dieback and acute oak decline (AOD) are considered two of the biggest threats for the ash and oak forests in Europe [6], [7]. Ash dieback was introduced to Europe in 1992 and in the United Kingdom (UK) in 2012 [8]. Only a minority of European ash trees $(\approx 5 \%)$ have resistance to ash dieback and it is estimated that a high proportion of trees will be damaged or die in the next 10-15 years in Scotland [9]. In addition to that, AOD is spreading rapidly in the UK forests during the last decade [7]. AOD is a bacterial infection that affects oak trees. Its unique characteristic compared to other fungi is that AOD is a very aggressive and fast-progressing agent that can lead to tree mortality within few years [10], [11].

Diagnosing the aforementioned diseases is particularly difficult due to the fact that early symptoms often affect the inner core without altering the appearance of the tree [13]. Decay inside the tree-trunk are robust indicators of the health status of a tree [13]. Tree decay are usually hollow, or liquid filled (for AOD [7]) and they are cylindrical-like targets aligned with the main axis of the trunk with arbitrary center and sizes [13]. Detecting tree decay and especially monitoring them in a dynamic manner is of great diagnostic importance and can vastly assist on preventing and containing potential threats. The most direct method to detect decay is coredrilling. It is apparent that this approach has severe practical limitations and it can potentially cause an irreversible damage to the tree [14]. Consequently, a wide range of geophysical non-destructive approaches have been suggested as potential solutions to this problem [15]-[21]. Electrical resistivity tomography (ERT) [17] and ultrasound tomography [21] are considered the most mainstream approaches while CT-scans [21], although accurate, have limited forestry applications due to operational constrains in the field. In the same context, microwave tomography using bespoke antenna systems with multiple transmitters and receivers have shown promising results on detecting tree decay [22], nonetheless, operational constrains makes it difficult to be applied in large scale forestry applications.

Common offset GPR is a -relatively- cheap and practical methodology with minimum operational and computational requirements that has been successfully applied in a wide range of sensing problems [28]. This makes it suitable for large scale forestry applications and has been suggested for mapping the root patterns as well as for evaluating the moisture content of the soil [23]-[26]. Regarding tree-trunk acquisitions, circular measurements is the usual survey configuration [27] due to the fact that the decay extend along the main axis of the trunk. Traditional GPR processing has been developed for surface measurements along clinical straight lines [27]. Consequently, typical GPR processing is not valid when the measurements take place along a complex tree trunk. This paper aims at providing a robust processing framework, fine-tuned for surveys along irregular closed curves. Through numerical and real experiments, strong indications are given that support the premise that the suggested scheme can accurately reconstruct tree-decay and can be used as a diagnostic tool for early detection of tree diseases. 
TABLE I

The Extended Debye Properties of the Tree Layers [33]

\begin{tabular}{rccccc}
\hline Name & $W C$ & $\epsilon_{\infty}$ & $\Delta \epsilon$ & $\sigma\left(\Omega^{-1} m^{-1}\right)$ & $t_{0}(\mathrm{sec})$ \\
\hline Cabdium layer & $70 \%$ & 9 & 43 & 1 & $9.23 \mathrm{e}-12$ \\
Outer Sapwood & $30 \%$ & 6.1 & 12.36 & 0.033 & $9.23 \mathrm{e}-12$ \\
Inner Sapwood & $25 \%$ & 5.9 & 9.66 & 0.02 & $9.23 \mathrm{e}-12$ \\
Rings & $10 \%$ & 5.4 & 3.1 & 0.0083 & $9.23 \mathrm{e}-12$ \\
Heartwood & $5 \%$ & 5.22 & 1.43 & 0.005 & $9.23 \mathrm{e}-12$ \\
Bark & $0 \%$ & 5 & 0 & 0 & $9.23 \mathrm{e}-12$ \\
\hline
\end{tabular}

\section{Distance to CoORdinates}

Typical GPR transducers employ a wheel-measuring device that effectively associates each measurement with its corresponding ordinate. This approach is valid when the survey takes place in a straight line. When the measurements are taken on an irregular closed curve, associating distance to coordinates is not a trivial task [29], [30]. Finding an accurate positioning method based on wheel-measurements is of great importance if commercial systems are to be used for evaluating the internal structure of the tree.

To that extend, the arc length parametarization [29], [30] is numerically calculated along the shape of the tree. Through this approach, the underlying relationship between the resulting distance from the measuring device and the coordinates for each measurement $M_{i}=\left\{\left(p_{i}, q_{i}\right) \mid i=1,2,3,4 \ldots n\right\}$, (where $n$ is the number of A-Scans) is evaluated. The shape of the curve is initially discretised using a sparse $m$ number of points $\left\{\left(x_{i}, y_{i}\right) \mid x_{i}, y_{i} \in \mathbb{R} \wedge i=1,2,3 \ldots m\right\}$. We then express both $x$ and $y$ in a parametric form using spline interpolation. The common parameter used to express $x(t)$ and $y(t)$ is $t \in[0,1]$. Notice that both $x(t)$ and $y(t)$ are now continuous and differentiable functions that map the curve of interest. Subsequently, the vector $\vec{R}=\langle x(t), y(t)\rangle$ is defined. The latter can be used to estimate the distance of every measurement along the curve from a reference point

$$
s(\tau)=\int_{0}^{\tau}\left\|\frac{d \vec{R}}{d t}\right\| d t=\int_{0}^{\tau} \sqrt{\left(\frac{d x(t)}{d t}\right)^{2}+\left(\frac{d y(t)}{d t}\right)^{2}} d t
$$

The integral in (1) is evaluated $v$ times numerically for different number of $\tau \in[0,1]$ and a set of points $\left(\left\{t_{j}, s_{j} \mid j=\right.\right.$ $1,2,3 \ldots v\})$ is obtained. Lastly, a spline interpolation is executed to map $t(s)$ in a continuous manner. The continuous function $t(s)$ can estimate the parametric variable $t$ for every distance from a reference point (along the curve). Lastly, the coordinates can be expressed with respect to distance via $x(t(s), y(t(s)))$.

\section{Processing Framework}

A typical tree-trunk consists of five layers (bark, cabdium, inner sapwwod, outer sapwood and heartwood) with different width and dielectric properties. The layered structure of the tree trunk gives rise to repetitive reflections that corrupt the resulting B-Scan. In order to remove the ringing noise, a
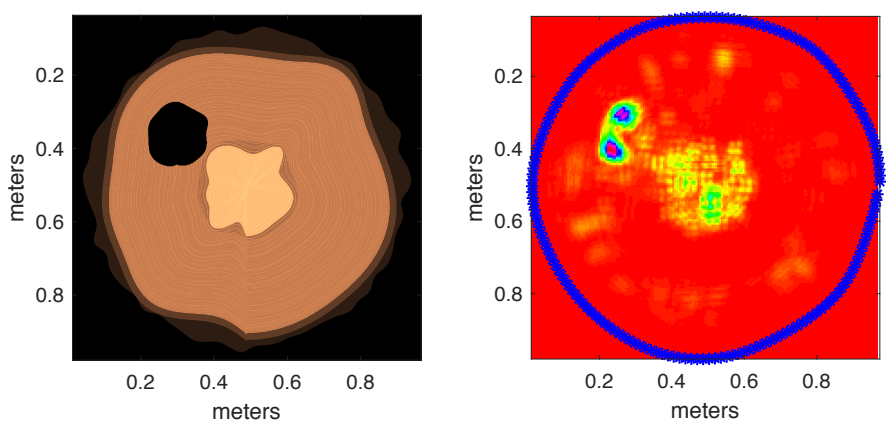

Fig. 1. Left: The simulated hardwood. Right: The reconstructed image after applying the suggested processing framework for $\epsilon=18$.
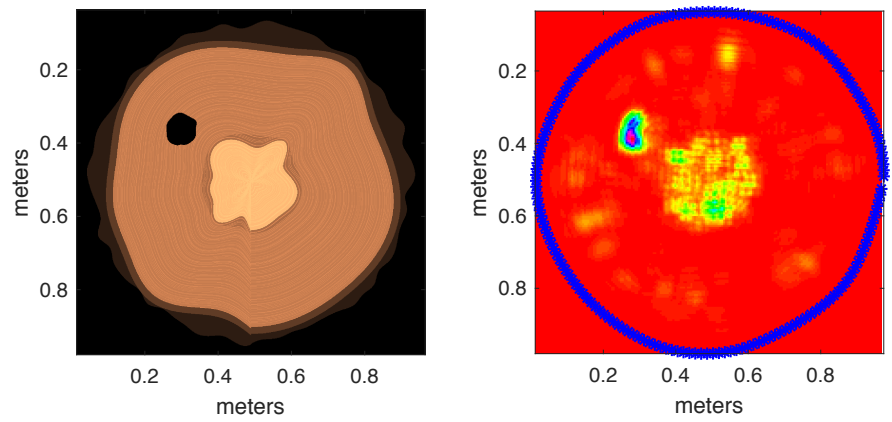

Fig. 2. Similar to Fig. 1 with a smaller tree decay.

singular value decomposition (SVD) filter [31] is applied prior to a linear gain and time-zero correction [33].

In the presence of a cylindrical target at $\left\langle x_{w}, y_{w}\right\rangle$ with radius $E$, the reflection arrival time will be equal to [33]

$$
t(s)=\left(\left\|\vec{R}(s)-\left\langle x_{w}, y_{w}\right\rangle\right\|-E\right) \frac{\sqrt{\epsilon}}{c_{0}}
$$

where $\epsilon$ is the relative bulk permittivity of the tree and $c_{0}$ is the speed of light. Notice that for line measurements, equation (2) represents a hyperbola. In the case of complex closed curves, the reflection arrival times give rise to complex patterns that are related to the size and the center of the target, the bulk permittivity of the tree and the shape of the tree. Similar to [33], the average permittivity $\epsilon$ is estimated from the twoway travel time from the opposite side of the trunk. A Perfect Conductor (PEC) is used to enhance the reflection.

Equation (2) for a given bulk permittivity $\epsilon$ is going to be used in order to apply a "hyperbola" summation for different point targets $x_{w}, y_{w}$. Initially, the processed B-Scan is expressed as a $2 \mathrm{D}$ function $B(t, s)$ related to time $t$ and distance $s$ from a given reference point. Substituting (2) to $B(t, s)$ and evaluating the integral across the curve of measurements results to

$L\left(x_{w}, y_{w}\right)=\int_{0}^{S} B\left(\left(\left\|\vec{R}(s)-\left\langle x_{w}, y_{w}\right\rangle\right\|-E\right) \frac{\sqrt{\epsilon}}{c_{0}}, s\right) d s$ 

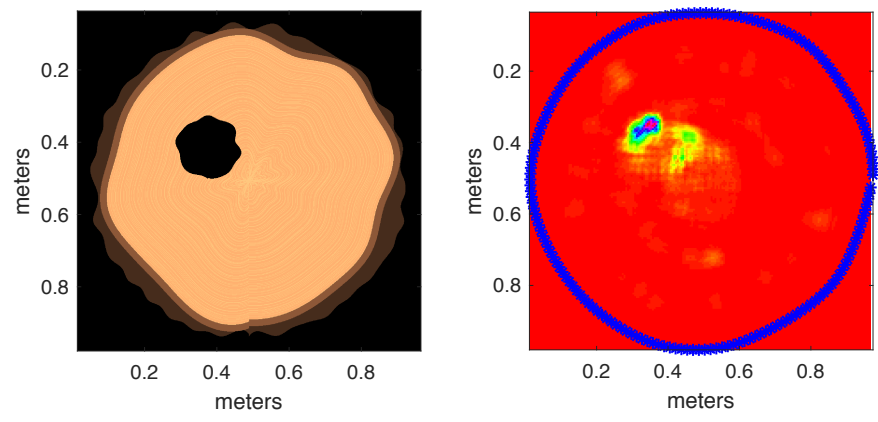

Fig. 3. Left: The simulated softwood. Right: The reconstructed image after applying the suggested processing scheme for epsilon $=18$.

Similar to typical Kirchhoff migration, a regularization term is applied here which reduces the artifacts arising from late reflections

$$
L\left(x_{w}, y_{w}\right)=\left(\min _{\forall s \in[0 S]} D(s)^{2}\right) \cdot \int_{0}^{S} \frac{B(D(s)-E, s)}{D(s)^{2}} \frac{\sqrt{\epsilon}}{c_{0}} d s
$$

where $D(s)=\left\|\vec{R}(s)-\left\langle x_{w}, y_{w}\right\rangle\right\|$. The regularization term represses the contributions of the later reflections that are often not associated with the investigated point $\left(x_{w}, y_{w}\right)$. The integral in (4) is evaluated numerically for different $\left(x_{w}, y_{w}\right)$ that lie inside the domain of interest and a 2D variable is created $M(x, y)$ which represents the migrated image. Subsequently, the matrix $M(x, y)$ is squared $T=M(x, y)^{2}$ in an effort to enhance the differences between targets and no targets. Lastly, the resulting image $T(x, y)$ is smoothed (Gaussian filter) in order to eliminate any high frequency components.

\section{NUMERICAL EXPERIMENTS}

A series of numerical experiments have been conducted in order to evaluate the ability of the suggested scheme on detecting tree decay. For the numerical simulations we have used gprMax [34], [35] an open source electromagnetic simulator that uses second order in both space and time finite difference time-domain (FDTD ) method [36]. In all the simulations the spacial step is $\Delta u=0.002, u \in\{x, y, z\}$ and the time-step equals with the Courant limit $\Delta t=3.81$ ps.

Dielectric properties of trees vary vastly based on the tree species, the season of measurements as well as the health status of the tree [37]. In general the relative permittivity is linearly proportional to the water content of the tree [38] and can reach values up to $\epsilon=40$ (for softwoods). Due to the importance of water to the overall relative permittivity of the tree, in the present numerical study we have followed the approach suggested in [33] in which the dispersive dielectric properties of the trunk are estimated based on its water content using a complex refractive index method (CRIM) [39]. The Debye dielectric properties used for each layer of the tree are shown in Table 1 [33]. The Debye expansions used in this paper resemblance the dielectric properties of a saturated hardwood or a semi-saturated softwood [33].

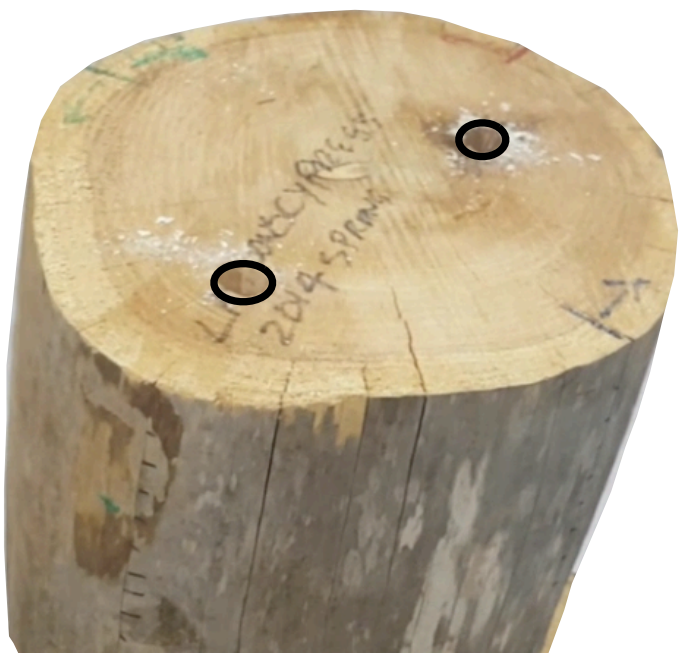

Fig. 4. The tree sample used for the laboratory experiment. The drilled holes are highlighted with black circles.

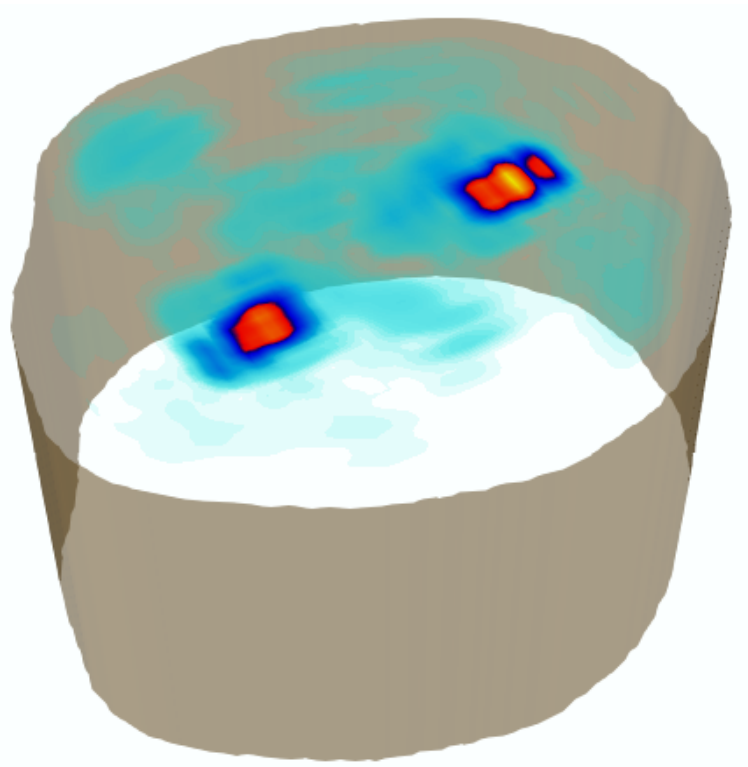

Fig. 5. The top horizontal slice of the reconstructed image for the laboratory experiment shown in Fig. 4.

In the first example, a hardwood is examined. Hardwoods -contrary to softwoods- have a distinct core named heartwood which is usually more dry compared to the sapwood [32]. The investigated hardwood has $\approx 0.5$ meters radius and the tree decay is a hollow chamber with relative permittivity $\epsilon=1$. The FDTD is excited using a numerical model of the commercial antenna GSSI $1.5 \mathrm{GHz}$ initially developed by [40] and later on modified by [41]. A bulk permittivity $\epsilon=18$ is used during the interpretation. Figure 1 illustrates both the synthetic model as well as the reconstructed image. It is evident that the proposed algorithm manages to accurately reconstruct both the tree decay as well as the inner heartwood of the investigated 


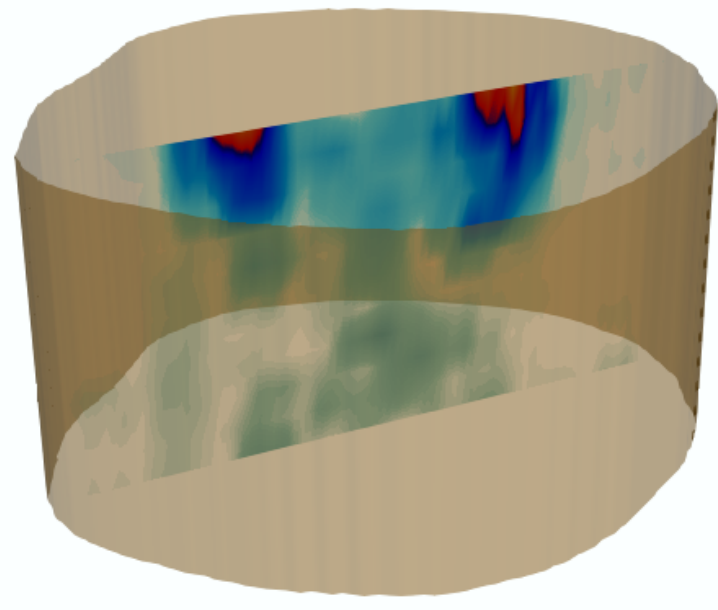

Fig. 6. The profile of the reconstructed decay for the laboratory experiment shown in Fig. 4.

hardwood. In the second numerical experiment, an early decay is implemented to the same host medium. Fig. 2 illustrates the simulated trunk as well as the reconstructed image. It is apparent that the investigated early decay has been accurately reconstructed indicating the potential of the current scheme as a diagnostic tool for early-stage tree diseases. The last numerical example is illustrated in Fig. 3. The ability of GPR to detect decay in softwoods (trunk without heartwood) is examined. As it is shown in Fig. 3, the proposed methodology performs equally well both for softwoods and hardwoods. Notice that the same processing scheme has been applied for all the aforementioned numerical examples without tuning it for each case study individually.

\section{LABORATORY MEASUREMENTS}

The proposed methodology is now tested in real measurements collected at The Faringdon Centre - Non-destructive Testing Centre, University of West London (UWL). The tree sample used in the current experiment is shown in Fig. 4. Two cylindrical holes $(\approx 1 \mathrm{~cm}$ radius and $\approx 4 \mathrm{~cm}$ depth$)$ were drilled in an effort to create artificial decay. The main axis of the cylinders are parallel to the main axis of the tree sample. Measurements were taken using the Aladdin $2 \mathrm{GHz}$ hand-held antenna from IDS GeoRadar (Part of Hexagon). In an effort to reconstruct a 3D image of the internal structure of the tree, circular measurements took place in slices with $2 \mathrm{~cm}$ distance from each other. The bulk permittivity of the tree was estimated $\epsilon=4$. The drilled holes were filled with saturated sawdust in an effort to simulate liquid filled cavities (a typical symptom of AOD). Figures 5 and 6 show the reconstructed decay. Both of the decay have been accurately detected and their length have been sufficiently estimated.

\section{CONCLUSIONS}

Through numerical and real experiments we have supported the premise that ground penetrating radar (GPR) has the potential to become a robust detection tool for early decay in tree-trunks. A novel processing scheme consisting of a singular value decomposition (SVD) filter for removing the ringing noise and the suggested modified Kirchhoff migration is proven very stable and accurate for the investigated case studies. The proposed framework can be applied in a straightforward manner using any commercial GPR system with minimum computational and operational requirements. This makes it particularly appealing for large-scale forestry applications and is a step forward to commercialization of GPR as a diagnostic tool for tree diseases.

\section{ACKNOWLEDGMENTS}

The authors would like to express their sincere thanks and gratitude to the following trusts, charities, organizations and individuals for their generosity in supporting this project: Lord Faringdon Charitable Trust, The Schroder Foundation, Cazenove Charitable Trust, Ernest Cook, Sir Henry Keswick, Ian Bond, P. F. Charitable Trust, Prospect Investment Management Limited, The Adrian Swire Charitable Trust, The John Swire 1989 Charitable Trust, The Sackler Trust, The Tanlaw Foundation and The Wyfold Charitable Trust.

This paper is dedicated to the memory of Jonathon West, a friend, a colleague, a forester, a conservationist and an environmentalist who died following an accident in the woodland that he loved.

\section{REFERENCES}

[1] Y. Pan, R. A. Birdsey, J. Fang, R. Houghton, P. E. Kauppi, W. A., Kurz, [...] D. Hayes, "A large and persistent carbon sink in the world's forests," Science, vol. 19, pp. 988-993, 2011.

[2] R. J. Mitchell, J. K. Beaton, P. E., Bellamy, A. Broome, J. Chetcutti, S. Eaton [...], S. woodward, "Ash dieback in the UK: A review of the ecological and conservation implications and potential management options," Biological Conservation, ovl. 175, pp. 95-109, 2014.

[3] Green Paper on Forest Protection and Information in the EU: Preparing forests for climate change, SEC, 2010.

[4] Feasibility Study on Means of Combating Forest Dieback in the European Union, Technical Report, BFG, EFI, 2007.

[5] C. Potter, T. Harwood, J. Knight, I. Tomlinson, "Learning from history, predicting the future: the UK Dutch elm disease outbreak in relation to contemporary disease threats," Philosophical Transactions of THe Royal Society, vol. 366, pp. 1966-1974, 2011.

[6] M. McMullan, M. Rafiqi, G. Kaithakottil, D. J., Clavijo, L. Bilham, E. Orton [...], M. D. Clark, "The ash dieback invasion of Europe was founded by two genetically divergent individuals," Nature Ecology and Evolution, vol. 2, pp. 1000-1008, 2018.

[7] N. Brown, D. J.G. Inward, M. Jeger and S. Denman, "A review of Agrilus biguttatus in UK forests and its relationship with acute oak decline," Forestry: An International Journal of Forest Research, vol. 88, no. 1, pp. 53-63, 2015.

[8] S. Papic, R. Longauer, I. Milenkovic, J. Rozsypalek, "Genetic predispositions of common ash to the ash dieback caused by ash dieback fungus," GENETIKA, vol. 25, no. 1, pp. 221-229, 2018.

[9] R. Worrell, An Assesment of The Potential Impacts of Ash Dieback in Scotland, Commisioned by Forestry Commission Scotland, 2013.

[10] N. Brown, Epidemiology of acute oak decline in Great Britain, $\mathrm{PhD}$ thesis submitted at Imperial College London, 2014.

[11] N. Brown, D. J.G. Inward, M. Jeger and S. Denman, "A review of Agrilus biguttatus in UK forests and its relationship with acute oak decline," Forestry: An International Journal of Forest Research, vol. 88, no. 1, pp. 53-63, 2015. 
[12] S. Denman, N. Brown, S. Kirk, M. Jeger and J. Webber, ”A description of the symptoms of Acute Oak Decline in Britain and a comparative review on causes of similar disorders on oak in Europe," Forestry: An International Journal of Forest Research, vol. 87, no. 4, pp. 535-551, 2014.

[13] W. C. Shortle, K. R. Dudzik, Wood Decay in Living and Dead Trees: A Pictorial Overview, U.S. FOREST SERVICE, 2012.

[14] J. Jezova, L. Mertens and S. Lambot, "Ground-penetrating radar for observing tree trunks and other cylindrical objects," Constraction and Building Materials, vol. 123, pp. 214-225, 2016.

[15] L. Costello and S. Quarles, "Detection of wood decay in blue gum and elm: An evaluation of the Resistograph and the portable drill," Journal of Arboriculture, vol. 25, pp. 311-317, 1999.

[16] S. A., Hagrey, "Electrical resistivity imaging of tree trunks," Near Surface Geophysics, vol. 4, pp. 179-187, 2006.

[17] G. Deflorio, S. Fink, and F. W. Schwarze, "Detection of incipient decay in tree stems with sonic tomography after wounding and fungal inoculation," Wood Science and Technology, vol. 42, pp. 117-132, 2008.

[18] A. Catena, "Thermography shows damaged tissue and cavities present in trees," Nondestructive Characterization of Materials, vol. 11, pp. 515522.

[19] A. Catena, "Thermography reveals hidden tree decay," Arboricultural Journal vol. 27, pp. 27-42, 2003.

[20] A. Catena, and A. G. Catena, "Overview of thermal imaging for tree assessment," Arboricultural Journal, vol. 30, pp. 259-270, 2008.

[21] Q. Wei, B. Leblon, and L. A. Rocque, "On the use of X-ray computed tomography for determining wood properties: a review," Can. J. For. Res. vol. 41. pp. 2120-2140, 2001.

[22] F. Boero, A. Fedeli, M. Lanini, M. Maffongelli, R. Monleone, M Pastorino, A. Randazoo, A. Slvade and A. Sansalone, "Microwave Tomography for the Inspection of Wood Materials: Imaging System and Experimental Results," IEEE Transactions on Microwave Theory and Techniques, vol. 66, no. 7, pp. 3497-3510, July 2018.

[23] G. Nicolotti, L.V. Socco, R. Martinis, A. Godio, L. Sambuelli, ”Application and comparison of three tomographic techniques for detection decay in trees", J. Arboric. vol. 29, pp. 66-78, 2003.

[24] H. Lorenzo, V. Prez-Gracia, A. Novo, J. Armesto, "Forestry applications of ground-penetrating radar," For. Syst., vol. 19, pp. 5-17, 2010.

[25] S. A. Al Hagrey, "Geophysical imaging of root-zone, trunk, and moisture heterogeneity," J. Exp. Bot. vol. 58, pp. 839-854, 2007.

[26] L. Lantini, R. Holleworth, D. Egyir, I. Giannakis, F. Tosti, A. Alani, , Use of ground penetrating radar for assessing interconnections between root systems of different matured tree species," in Proc. MetroArchaeo, 2018, Italy.

[27] J. Jezova, J. Harou and S. Lambot, "Reflection waveforms occurring in bistatic radar testing of columns and tree trunks," Construction and Building Materials, vol. 174, pp. 388-400, 2018.

[28] D. J. Daniels, Ground Penetrating Radar, 2nd ed. London, U.K.: Institution of Engineering and Technology, 2004.

[29] R. J. Sharpe, R. W. Thorpe, "Numerical Method for Extracting an Arc Length Parameterization from Parametric Curves", Computer Aided Design, vol. 12, no. 2, pp. 79-81, March 1982.

[30] B., Guenter and R. Parent, "Computing the arc length of parametric curves,-IEEE Comp. Graph. Appl. , vol. 10, no. 3, pp. 72-78, 1990.

[31] H. Kim, S. J. Cho, and M. J. Yi, "Removal of ringing noise in GPR data by signal processing," Geosci. J. , vol. 11, pp. 75-81, Mar. 2007.

[32] C. Humphries, B. Press and D. Sutton, Guide to Trees of Britain and Europe, Philip's, London, 2006.

[33] I. Giannakis, F. Tosti, L. Lantini, A. Alani, "Health Monitoring of Tree-Trunks Using Ground Penetrating Radar,' IEEE Transactions on Geoscience and Remote Sensing, Under review.

[34] C. Warren, A. Giannopoulos, and I. Giannakis, "gprMax: Open source software to simulate electromagnetic wave propagation forGround Penetrating Radar,' Comput. Phys. Commun. , vol. 209, pp. 163170, Dec. 2016.

[35] C. Warren, A. Giannopoulos, A. Gray, I. Giannakis, A. Patterson, L. Wetter, A. Hamrah, "A CUDA-based GPU engine for gprMax: Open source FDTD electromagnetic simulation software," Computer Physics Communications, Early Access, 2018,

[36] A. Taflove and S. C. Hagness, Computational Electrodynamics, the Finite-Difference Time-Domain Method, 2nd ed. Norwood, MA, USA: Artech House, 2000.
[37] T. Douglas, Effective Dielectric Constants of Foliage Media, Rome Air Development Center Air Force Systems Command Grifflss Air Force Base, Rome, 1990.

[38] M. G. Broadhurst, "Complex Dielectric Constants and Dissipation Factor of Foliage," NBS Report no. 9592, NBS project 3110107, US Naval Ordnance Laboratory, October 1970.

[39] R. J. Birchak, C. G. Gardner, E. J. Hipp, and M. J. Victor, "High dielectric constant microwave probes for sensing soil moisture," in Proc. of the IEEE, vl. 62, no. 1, pp 93-98, 1974.

[40] C. Warren and A. Giannopoulos, "Creating finite-difference time-domain models of commercial ground-penetrating radar antennas using Taguchis optimisation method," Geophysics, vol. 76, no. 2, pp. G37-G47, Apr. 2011.

[41] I. Giannakis, A. Giannopoulos and C. Warren, "Realistic FDTD GPR Antenna Models Optimized Using a Novel Linear/Nonlinear FullWaveform Inversion," IEEE Trans. on Geoscience and Remote Sensing, Early Access, 2018. 